\title{
Magnetic dynamics of simple collective modes in a two-sphere plasma model
}

\author{
Hanno Essén \\ Department of Mechanics, KTH \\ SE-100 44 Stockholm, Sweden
}

(Dated: 2005 November)

\begin{abstract}
A plasma blob is modeled as consisting of two homogeneous spheres of equal radius and equal but opposite charge densities that can move relative to each other. Relative translational and rotational motion are considered separately. Magnetic effects from the current density caused by the relative motion are included. Magnetic interaction is seen to cause an inductive inertia. In the relative translation case the Coulomb attraction, approximately a linear force for small amplitudes, causes an oscillation. For a large number of particles the corresponding oscillation frequency will not be the Langmuir plasma frequency, because of the large inductive inertia. For rotation an external magnetic field is included and the energy and diamagnetism of the plasma in the model is calculated. Finally it is noted how the neglect of resistivity is motivated by the results.
\end{abstract}




\section{INTRODUCTION}

In order to study qualitative effects of magnetism in plasma dynamics a very simple model is introduced. Two overlapping homogeneous spheres of equal radii, and of equal but oppositely signed charge densities, are assumed to move, relative to each other, with negligible dissipation (resistivity) under the influence of electric and magnetic interaction. The neglect of dissipation is motivated at the end. We first treat relative translation (oscillation) and then relative rotation.

Many years ago Tonks and Langmuir [1] carefully derived an equation of motion for a collective translational motion of the electrons relative to the positive ions. Their derivation seems to indicate that there should be a universal frequency for this mode,

$$
\omega_{\mathrm{p}}=\sqrt{\frac{n e^{2}}{m}},
$$

the plasma frequency, depending only on the electron number density $n$. If a large number of electrons move relative to the positive ions one gets a large current and thus it seems as if magnetic effects should affect the result. Bohm and Pines [2] studied the influence of magnetic interaction on plasma modes but they did not come up with any explicit correction to $\omega_{\mathrm{p}}$. In the textbook by Goldston and Rutherford [3] the absence of magnetic effects is said to be due to a displacement current that compensates for the electron current. In this article the problem is approached from a very fundamental starting point: the relevant Lagrangian density. The conclusion is that the frequency is lowered by the large inductive inertia [see Eq. (21)].

We then study the relative rotation of the two charged spheres. If magnetic interaction is neglected the kinetic energy is simply determined by angular momentum and moment of inertia. When magnetic interaction is included the kinetic energy for a given angular momentum is much smaller. The reason for this is again that the effective moment of inertia will be dominated by inductive inertia. By adding an external magnetic field to the model we can calculate the response of our model plasma and it turns out to be diamagnetic. 


\section{SEPARATION OF OVERALL TRANSLATION AND ROTATION}

The kinetic energy of any system of particles

$$
T=\sum_{i} \frac{1}{2} m_{i} \boldsymbol{v}_{i}^{2}
$$

can be written

$$
T=\frac{1}{2} M \boldsymbol{V}^{2}+\frac{1}{2} \Omega \mathrm{J} \Omega+T^{\prime}
$$

where $M$ is total mass, $\mathrm{J}$ is the (instantaneous) inertia tensor, $\boldsymbol{V}$ center of mass velocity, and $\boldsymbol{\Omega}$ is a well defined average angular velocity [4, 5]. $\boldsymbol{V}$ is chosen so that $\boldsymbol{p}=M \boldsymbol{V}$ is the total momentum of the system and $\boldsymbol{\Omega}$ so that $\boldsymbol{L}=\mathrm{J} \boldsymbol{\Omega}$ is the total (center of mass) angular momentum. $T^{\prime}$ is the kinetic energy of the particles relative to the system that moves with the center of mass velocity and rotates with the average angular velocity. We call this the co-moving system. One can introduce generalized coordinates so that there are six degrees freedom describing center of mass position and of average angular orientation, while $T^{\prime}$ depends on the remaining $3 N-6$ generalized coordinates.

Now consider a blob of plasma that consists of two spheres of particles, one of positively, and one of negatively charged particles. For spheres the inertia tensor $\mathrm{J}$ can be replaced by a single moment of inertia $J$. The total kinetic energy $T$ is the sum of the kinetic energy, $T_{1}$, of the positive particles, and of the kinetic energy, $T_{2}$, of the negative charges.

We first perform the transformation above to the co-moving systems separately for the positive and the negative particles. This gives,

$$
T=\frac{1}{2} M_{1} \boldsymbol{V}_{1}^{2}+\frac{1}{2} M_{2} \boldsymbol{V}_{2}^{2}+\frac{1}{2} J_{1} \Omega_{1}^{2}+\frac{1}{2} J_{2} \Omega_{2}^{2}+T_{1}^{\prime}+T_{2}^{\prime},
$$

for the total kinetic energy, see Fig. 1, In a second step we then introduce the co-moving system for the total system. We thus introduce,

$$
\begin{array}{cc}
M=M_{1}+M_{2}, & J=J_{1}+J_{2}, \\
\mu=M_{1} M_{2} / M, & I=J_{1} J_{2} / J,
\end{array}
$$

total mass and reduced mass as well as total moment of inertia and reduced moment of inertia. In terms of these one finds,

$$
\begin{aligned}
\boldsymbol{V}=\left(M_{1} \boldsymbol{V}_{1}+M_{2} \boldsymbol{V}_{2}\right) / M & \boldsymbol{V}_{1}=\boldsymbol{V}+M_{2} \boldsymbol{v} / M \\
\boldsymbol{v}=\boldsymbol{V}_{1}-\boldsymbol{V}_{2} & \boldsymbol{V}_{2}=\boldsymbol{V}-M_{1} \boldsymbol{v} / M
\end{aligned}
$$


for the total center of mass velocity $\boldsymbol{V}$, and relative velocity $\boldsymbol{v}$, of the two spheres. Finally,

$$
\begin{aligned}
\boldsymbol{\Omega}=\left(J_{1} \boldsymbol{\Omega}_{1}+J_{2} \boldsymbol{\Omega}_{2}\right) / J & \boldsymbol{\Omega}_{1}=\boldsymbol{\Omega}+J_{1} \boldsymbol{\omega} / J \\
\boldsymbol{\omega}=\boldsymbol{\Omega}_{1}-\boldsymbol{\Omega}_{2} & \boldsymbol{\Omega}_{2}=\boldsymbol{\Omega}-J_{2} \boldsymbol{\omega} / J
\end{aligned}
$$

gives the total average angular velocity $\boldsymbol{\Omega}$, and the relative angular velocity $\boldsymbol{\omega}$, of the two oppositely charged spheres. In terms of these quantities we get the expression,

$$
T=\frac{1}{2} M \boldsymbol{V}^{2}+\frac{1}{2} \mu \boldsymbol{v}^{2}+\frac{1}{2} J \Omega^{2}+\frac{1}{2} I \boldsymbol{\omega}^{2}+T^{\prime},
$$

for the total kinetic energy of our two-sphere system. The degrees of freedom in $T^{\prime}$ are assumed to be random and not to produce any net charge or current density. They will be ignored henceforth.

\section{LAGRANGIAN INCLUDING MAGNETIC INTERACTIONS}

Maxwell's equations and the equations of motion for the charged particles with the Lorentz force, can all be derived from a single Lagrangian via the variational principle [6]. The Lagrangian has three parts, particle, interaction, and field contributions. If radiation is neglected the field does not have independent degrees of freedom, but is determined by particle positions and velocities. Using the non-relativistic form for the kinetic energy one then gets,

$$
L=\sum_{i}\left(\frac{1}{2} m_{i} \boldsymbol{v}_{i}^{2}+\frac{q_{i}}{2 c} \boldsymbol{v}_{i} \cdot \boldsymbol{A}\left(\boldsymbol{r}_{i}\right)-\frac{q_{i}}{2} \phi\left(\boldsymbol{r}_{i}\right)\right)
$$

where,

$$
\phi(\boldsymbol{r}, t)=\sum_{i} \frac{q_{i}}{\left|\boldsymbol{r}-\boldsymbol{r}_{i}\right|},
$$

and

$$
\boldsymbol{A}(\boldsymbol{r}, t)=\sum_{i} \frac{q_{i}\left[\boldsymbol{v}_{i}+\left(\boldsymbol{v}_{i} \cdot \boldsymbol{e}_{i}\right) \boldsymbol{e}_{i}\right]}{2 c\left|\boldsymbol{r}-\boldsymbol{r}_{i}\right|} .
$$

Here the position and velocity vectors of the particles are $\boldsymbol{r}_{i}$ and $\boldsymbol{v}_{i}$ respectively, $m_{i}$ and $q_{i}$ their masses and charges, and $\boldsymbol{e}_{i}=\left(\boldsymbol{r}-\boldsymbol{r}_{i}\right) /\left|\boldsymbol{r}-\boldsymbol{r}_{i}\right|$ (Darwin [7], Jackson [8], Schwinger et al. [9], Essén [10, 11]). The vector potential here is in the Coulomb gauge, and this essentially means that all velocity dependence of the interaction appears in the magnetic part, leaving the Coulomb interaction energy in its static form. 
When the expressions (13) and (14) are inserted into equation (12) one finds infinite contributions from self-interactions. When these are discarded, so that each particle only interacts with the field from the others, one obtains

$$
L=\sum_{i} \frac{1}{2} m_{i} \boldsymbol{v}_{i}^{2}+\sum_{i<j} \frac{q_{i} q_{j}}{r_{i j}} \frac{\left[\boldsymbol{v}_{i} \cdot \boldsymbol{v}_{j}+\left(\boldsymbol{v}_{i} \cdot \boldsymbol{e}_{i j}\right)\left(\boldsymbol{v}_{j} \cdot \boldsymbol{e}_{i j}\right)\right]}{2 c^{2}}-\sum_{i<j} \frac{q_{i} q_{j}}{r_{i j}},
$$

where now $r_{i j}$ is the distance between particles $i$ and $j$ and $\boldsymbol{e}_{i j}$ is the unit vector pointing from $i$ to $j$. This is the so called Darwin Lagrangian [7] for the system. We can write it,

$$
L=T+L_{\mathrm{mag}}-\Phi
$$

where, $T$, is kinetic energy and, $\Phi$, the Coulomb electric interaction energy. The magnetic part can also be written

$$
L_{\mathrm{mag}}=\sum_{i} \frac{q_{i}}{2 c} \boldsymbol{v}_{i} \cdot \boldsymbol{A}\left(\boldsymbol{r}_{i}\right)=\frac{1}{2 c} \int \boldsymbol{j}(r) \cdot \boldsymbol{A}(r) \mathrm{d} V .
$$

Here it is important the the vector potential is divergence free $(\nabla \cdot \boldsymbol{A}=0$, Coulomb gauge). The Darwin Lagrangian thus includes both electric and magnetic interactions between the particles and is valid in when radiation can be neglected.

\section{RELATIVE TRANSLATIONAL MOTION}

The Coulomb interaction, $\Phi$, between two overlapping charged spheres is calculated in Appendix A, The magnetic interaction between two charged spheres in relative translational motion is calculated in Appendix B for the case of small displacement of centers of the spheres $(r \ll R)$. Keeping only the quadratic term, one finds,

$$
L_{\mathrm{rel}}=\frac{1}{2} \mu \boldsymbol{v}^{2}+\frac{4 Q^{2}}{10 R c^{2}} \boldsymbol{v}^{2}-\frac{1}{2} \frac{Q^{2}}{R^{3}} \boldsymbol{r}^{2} .
$$

Here $\boldsymbol{r}$ is the vector to center of the positive sphere from the center of the negative, so that $\dot{\boldsymbol{r}}=\boldsymbol{v}$. The center of mass motion decouples, and we assume that the random motions decouple. This is then the relevant Lagrangian for the relative collective translation. It can be written,

$$
L_{\mathrm{rel}}=\frac{1}{2} \mathcal{M} \boldsymbol{v}^{2}-\frac{1}{2} \mathcal{K} \boldsymbol{r}^{2}
$$

where,

$$
\mathcal{M}=\mu+\frac{4 Q^{2}}{5 c^{2} R} \approx N m\left(1+\frac{4}{5} \frac{N r_{\mathrm{e}}}{R}\right)
$$


and $\mathcal{K}=Q^{2} / R^{3}$. If we assume a proton-electron plasma we get $M_{1}=N m_{\mathrm{p}}, M_{2}=N m$, $\mu=N m_{\mathrm{p}} m /\left(m_{\mathrm{p}}+m\right) \approx N m$, and $Q^{2}=N^{2} e^{2}$. On the right hand side we have introduced, $r_{\mathrm{e}} \equiv \frac{e^{2}}{m c^{2}}$, the classical electron radius. Note that when $N r_{\mathrm{e}} / R \gg 1$ the effective mass $\mathcal{M}$ is entirely due to inductive inertia.

Clearly the Lagrangian (19) corresponds to an oscillating system with angular frequency $\omega_{0}=\sqrt{\mathcal{K} / \mathcal{M}}$. For this frequency we get explicitly,

$$
\omega_{0}^{2}=\frac{\frac{N e^{2}}{R^{3} m}}{1+\frac{4}{5} \frac{N r_{\mathrm{e}}}{R}} .
$$

If we introduce the dimensionless number,

$$
\nu \equiv N r_{\mathrm{e}} / R
$$

we see that for $\nu \ll 1$, one obtains, essentially, the Langmuir plasma frequency, $\omega_{\mathrm{p}}^{2} \propto n e^{2} / m$, see Eq. (1). If we reexpress the plasma frequency in terms of the classical electron radius [12], Eq. (21) can be written in the form,

$$
\omega_{0}^{2}=\frac{\nu}{1+\frac{4}{5} \nu} \frac{c^{2}}{R^{2}} .
$$

Thus, when the number of particles is large enough so that $\nu \gg 1$, this gives,

$$
\omega_{0}^{2}=\frac{5 c^{2}}{4 R^{2}}
$$

For this case the frequency turns out to depend on the size (radius) of the sphere, but not on the density.

\section{RELATIVE ROTATIONAL MOTION}

We now study pure rotational motion of the two charged spheres, about their coinciding centers of mass, but we include interaction,

$$
L_{\mathrm{e}}=\frac{1}{c} \int \boldsymbol{j}(\boldsymbol{r}) \cdot \boldsymbol{A}_{\mathrm{e}}(\boldsymbol{r}) \mathrm{d} V
$$

with a constant external magnetic field $\boldsymbol{B}=\nabla \times \boldsymbol{A}_{\mathrm{e}}$. Here $\boldsymbol{A}_{\mathrm{e}}=\frac{1}{2} \boldsymbol{B} \times \boldsymbol{r}$ is the vector potential of the external field. Starting from (11) and (16) we find that,

$$
L_{\mathrm{rot}}=\frac{1}{2} I \boldsymbol{\omega}^{2}+L_{\mathrm{mag}}+L_{\mathrm{e}},
$$


is the relevant Lagrangian for collective relative rotation.

The explicit calculations are sketched in Appendix C. One finds that $L_{\mathrm{mag}} \sim \boldsymbol{\omega}^{2}$ and that this term therefore contributes to the effective moment of inertia, just as it contributed to the effective mass, $\mathcal{M}$, in the translational case. The result can be written,

$$
L_{\mathrm{rot}}=\frac{1}{2} \mathcal{I} \boldsymbol{\omega}^{2}+\frac{Q R^{2}}{10 c} \boldsymbol{\omega} \cdot \boldsymbol{B}
$$

where,

$$
\mathcal{I}=I+\frac{2}{35} \frac{Q^{2} R}{c^{2}}=\frac{2}{5} N m R^{2}\left(1+\frac{1}{7} \frac{N r_{\mathrm{e}}}{R}\right) .
$$

We see that when $\nu=N r_{\mathrm{e}} / R \gg 1$ we can neglect the contribution from mass to the effective moment of inertia $\mathcal{I}$. In this limit therefore,

$$
\mathcal{I} \approx \frac{2}{35} \frac{Q^{2} R}{c^{2}}
$$

and there is essentially only inductive moment of inertia. We assume this below.

\section{PLASMA ENERGY AND DIAMAGNETISM}

In order to investigate the equation of motion we put $\boldsymbol{\omega}=\dot{\varphi} \boldsymbol{e}_{z}$ and $\boldsymbol{B}=B\left(\sin \theta \boldsymbol{e}_{x}+\right.$ $\left.\cos \theta \boldsymbol{e}_{z}\right)$. The Lagrangian then becomes

$$
L_{\mathrm{rot}}=\frac{1}{2} \mathcal{I} \dot{\varphi}^{2}+\frac{Q R^{2}}{10 c} \dot{\varphi} B \cos \theta
$$

In general when, $\partial L / \partial \varphi=0$, one finds that, $\dot{p}_{\varphi}=(\mathrm{d} / \mathrm{d} t)(\partial L / \partial \dot{\varphi})=0$. In our case this gives,

$$
p_{\varphi}=\frac{\partial L_{\mathrm{rot}}}{\partial \dot{\varphi}}=\mathcal{I} \dot{\varphi}+\frac{Q R^{2}}{10 c} B \cos \theta=\text { const. }
$$

If we assume that $\dot{\varphi}(t=0)=0$ when $B(t=0)=0$ we find that the constant is zero: $p_{\varphi}=0$. At all times we then find the relation,

$$
\dot{\varphi}(t)=\frac{7 R c}{4 Q} B(t) \cos \theta
$$

between angular velocity and the magnetic field.

To get the energy from $L(\varphi, \dot{\varphi})$ one calculates the Hamiltonian $H=p_{\varphi} \dot{\varphi}-L$. For the $L_{\text {rot }}$ of Eq. (30) one finds,

$$
H=\frac{1}{2 \mathcal{I}}\left(p_{\varphi}-\frac{Q R^{2}}{10 c} B \cos \theta\right)^{2} .
$$


Let us consider two special cases of this phase space energy of the plasma.

We first assume that the external field is zero $(B=0)$. The energy is then given by $E=H=p_{\varphi}^{2} / 2 \mathcal{I}$. Here $p_{\varphi}$ is the angular momentum of relative rotation. For a given value of this angular momentum the energy is thus much smaller when $\nu \gg 1$ than otherwise. This reflects the fact, repeatedly stressed by the author [10, 11, 13, 14], that for given momenta the phase space energy of a plasma is lower when there is net current than in the absence of net current.

Now consider instead the case $p_{\varphi}=0$. For simplicity we also assume $\theta=0$. One then finds that

$$
H\left(p_{\varphi}=0\right)=\frac{1}{2 \mathcal{I}}\left(\frac{Q R^{2}}{10 c} B\right)^{2}
$$

or, equivalently, using (29), that the energy as function of $B$ is given by,

$$
E(B)=\frac{7}{80} R^{3} B^{2}=\frac{21}{40}\left(\frac{4 \pi R^{3}}{3}\right) \frac{B^{2}}{8 \pi} .
$$

Note that here, $B^{2} / 8 \pi$, is the energy density of the field $B$ in our (gaussian) units. The energy is thus seen to grow quadratically with the applied magnetic field and our plasma spheres are strongly diamagnetic. Based on more detailed studies Cole [15] has also concluded that plasmas are diamagnetic. In our model plasma diamagnetism is seen to be closely related to the diamagnetism of superconductors, as discussed by Essén [16]: the external field induces a current that screens the external field and reduces it inside. In the absence of resistance this screening current persists.

\section{PLASMA RESISTIVITY}

Resistivity is completely neglected in the present model. It has been pointed out by Kulsrud [17], in his book on astrophysical plasmas, that the negligible resistivity of such plasmas is in fact closely connected with magnetic induction. In the present treatment magnetic induction appears in the form of an inductive inertia that appears naturally as the main physical parameter in the present model. As early as 1933 Frenkel [18] suggested that superconductivity is due to inductive inertia. Frenkel also conjectured that inductive inertia can lower the energy and cause a phase transition. He did not, however, make his ideas quantitative. The present model system gives Frenkel's ideas some quantitative backing. 
We note that for a collective momentum $p$ involving $N$ particles the kinetic energy will be $T=p^{2} /(2 m N)$, when magnetic interaction is neglected, see Eqs. (19-201). When the effect of magnetic interaction is included this becomes $T+E_{\mathrm{mag}}=p^{2} /(2 N m[1+4 \nu / 5])$ and we find that $T+E_{\text {mag }} \ll T$ when $\nu \gg 1$, assuming that $p$ remains constant. Collective modes are thus much more favorable thermodynamically when there is net current.

Plasma resistivity is normally treated by studying the scattering of individual charged particles. Even with this type of treatment fast electrons become, so called, runaway electrons and experience no resistance [19]. The present model indicates that resistivity can not be treated as resulting from the scattering of individual particles, since the collective motion of many charges leads to a large inductive non-local effect. All this points in the same direction, namely that plasmas need not be resistive, in agreement with our model treatment.

If resistivity had to be included the translational oscillation would become a damped oscillation and any circulating current would eventually cease, thereby making the diamagnetic response temporary. A more immediate limitation of our model is probably the fact that a current would cause pinching and this would lead to instabilities that deform of the spherical shape. Lynden-Bell [22] has studied the relativistically spinning charged sphere and finds that charge concentrates near the equator (as a result of pinching).

\section{CONCLUSIONS}

The model treated in this article is not particularly realistic. Instead it can be motivated as the simplest possible model within which one can study plasma phenomena associated with current, induction, and magnetic interaction energy, in a meaningful way. Hopefully it also has some novelty. In the literature one can find a fair amount of work on the radially oscillating plasma sphere (see e.g. Barnes and Nebel [20], Park et al. [21]), but not the modes treated here. A numerical study of a rotating convective plasma sphere, modelling a star, by Dobler et al. [23] shows how complicated more realistic models necessarily become.

The two-sphere model studied here is therefore valuable as a device for gaining insight into some very basic plasma phenomena. As we have seen the most basic of these is the dominance of inductive inertia in the effective mass $\mathcal{M}$ of Eq. (20), and the effective moment of inertia $\mathcal{I}$ of Eq. (28), when the number $N$ of participating charged particles is large enough. 
One notes that $\nu=N r_{\mathrm{e}} / R \approx 118$ for a typical laboratory plasma of density $n=10^{20} \mathrm{~m}^{-3}$ and of radius $R=1 \mathrm{~cm}$, assuming that all particles contribute to the collective mode. Finally the model also indicates how this large inductive inertia influences the energy of the plasma and how a plasma responds diamagnetically to an external magnetic field. 


\section{Appendices}

\section{Appendix A: Electrostatic interaction of two overlapping charged spheres}

The electrostatic potential from a spherically symmetric charge density, $\varrho(r)$, is given by,

$$
\phi(r)=4 \pi\left(\frac{1}{r} \int_{0}^{r} \varrho\left(r^{\prime}\right) r^{\prime 2} \mathrm{~d} r^{\prime}+\int_{r}^{\infty} \varrho\left(r^{\prime}\right) r^{\prime} \mathrm{d} r^{\prime}\right)
$$

For a homogeneous charged sphere of total charge $Q$ and radius $R$ with $\varrho=Q / \frac{4 \pi R^{3}}{3}$, for $r \leq R$ and zero for $r>R$, this gives,

$$
\phi(r)= \begin{cases}\frac{3 Q}{2 R}-\frac{Q r^{2}}{2 R^{3}} & \text { for } r<R \\ \frac{Q}{r} & \text { for } r \geq R,\end{cases}
$$

and there is a pure Coulomb potential outside the sphere.

Now consider a second, homogeneously charged, sphere of the same radius and of total charge $q$. If the distance $r$ between the centers of the two spheres is greater than $2 R$ the interaction energy is clearly given given by,

$$
\Phi(r)=\frac{q Q}{r}, \quad \text { for } r>2 R
$$

We will now investigate what this interaction energy is when the distance is smaller so that the two spheres intersect.

The interaction energy can be written

$$
\Phi(r)=\int \varrho_{q} \phi \mathrm{d} V
$$

where the volume integration is over the space occupied by the second sphere, where its charge density, $\varrho_{q}=q / \frac{4 \pi R^{3}}{3}$, is different from zero.

We do the integration using the slicing by concentric spheres depicted in Fig. 2. The volume element is then given by

$$
\mathrm{d} V=\pi \frac{\rho}{r}\left[R^{2}-(r-\rho)^{2}\right] \mathrm{d} \rho .
$$

When this is integrated between the limits $\rho=r-R$ and $\rho=r+R$ one should get the volume of the sphere and indeed and one easily checks that

$$
\int_{\rho=r-R}^{\rho=r+R} \mathrm{~d} V(\rho)=\frac{4 \pi R^{3}}{3} .
$$


To do the integral (A4) we have to split the integration range at $\rho=R$ since the function $\phi$ of (A2) since it changes character at that radius. This gives

$$
\Phi(r)=\varrho_{q}\left[\int_{\rho=r-R}^{\rho=R}\left(\frac{3 Q}{2 R}-\frac{Q \rho^{2}}{2 R^{3}}\right) \mathrm{d} V+\int_{\rho=R}^{\rho=r+R} \frac{Q}{\rho} \mathrm{d} V\right] .
$$

The integrals are elementary and the final result, for $r<2 R$, is,

$$
\Phi(r)=\frac{q Q}{160 R^{6}}\left(192 R^{5}-80 R^{3} r^{2}+30 R^{2} r^{3}-r^{5}\right) .
$$

If we assume small displacements, $r \ll R$, and put $q=-Q$, we find that,

$$
\Phi(r) \approx-\frac{6 Q^{2}}{5 R}+\frac{Q^{2} r^{2}}{2 R^{3}}
$$

so the potential is quadratic and there is there is a linear restoring radial force, $F=-k r$, with force constant, $k=Q^{2} / R^{3}$.

\section{Appendix B: Magnetic interaction of two charged spheres with relative translation}

Here we calculate $L_{\mathrm{mag}}$, the middle term of Eq. (15), for the two charged spheres with velocities $\boldsymbol{V}_{1}$ and $\boldsymbol{V}_{2}$ of Eqs. (7-8). This Darwin term in the Lagrangian gives explicitly,

$$
\begin{array}{r}
L_{\mathrm{mag}}=\frac{q_{1}^{2}}{4 c^{2}} \sum_{i} \sum_{j} \frac{\boldsymbol{V}_{1}^{2}+\left(\boldsymbol{V}_{1} \cdot \boldsymbol{e}_{i j}\right)^{2}}{\left|\boldsymbol{r}_{i}-\boldsymbol{r}_{j}\right|}+ \\
\frac{q_{1} q_{2}}{4 c^{2}} \sum_{i} \sum_{k} \frac{\boldsymbol{V}_{1} \cdot \boldsymbol{V}_{2}+\left(\boldsymbol{V}_{1} \cdot \boldsymbol{e}_{i k}\right)\left(\boldsymbol{V}_{2} \cdot \boldsymbol{e}_{i k}\right)}{\left|\boldsymbol{r}_{i}-\boldsymbol{r}_{k}\right|}+ \\
\frac{q_{2} q_{1}}{4 c^{2}} \sum_{k} \sum_{i} \frac{\boldsymbol{V}_{2} \cdot \boldsymbol{V}_{1}+\left(\boldsymbol{V}_{2} \cdot \boldsymbol{e}_{k i}\right)\left(\boldsymbol{V}_{1} \cdot \boldsymbol{e}_{k i}\right)}{\left|\boldsymbol{r}_{k}-\boldsymbol{r}_{i}\right|}+ \\
\frac{q_{2}^{2}}{4 c^{2}} \sum_{k} \sum_{l} \frac{\boldsymbol{V}_{2}^{2}+\left(\boldsymbol{V}_{2} \cdot \boldsymbol{e}_{k l}\right)^{2}}{\left|\boldsymbol{r}_{k}-\boldsymbol{r}_{l}\right|}
\end{array}
$$

for the magnetic interaction. Here the indices $i, j$ refer to the positive sphere and $k, l$ to the negative. The two terms (B2) and (B33) representing interactions between the two oppositely

charged spheres are clearly equal. If we denote the angle in (B1) between $\boldsymbol{V}_{1}$ and $\boldsymbol{r}_{i}-\boldsymbol{r}_{j}$ by $\theta_{i j}$, and similarly for ( $(\underline{\mathrm{B} 4})$, we thus get

$$
\begin{array}{r}
L_{\mathrm{mag}}=\frac{q_{1}^{2} V_{1}^{2}}{4 c^{2}} \sum_{i} \sum_{j} \frac{1+\cos ^{2} \theta_{i j}}{\left|\boldsymbol{r}_{i}-\boldsymbol{r}_{j}\right|}+\frac{q_{2}^{2} V_{2}^{2}}{4 c^{2}} \sum_{k} \sum_{l} \frac{1+\cos ^{2} \theta_{k l}}{\left|\boldsymbol{r}_{k}-\boldsymbol{r}_{l}\right|} \\
+\frac{q_{1} q_{2}}{2 c^{2}} \sum_{i} \sum_{k} \frac{\boldsymbol{V}_{1} \cdot \boldsymbol{V}_{2}+\left(\boldsymbol{V}_{1} \cdot \boldsymbol{e}_{i k}\right)\left(\boldsymbol{V}_{2} \cdot \boldsymbol{e}_{i k}\right)}{\left|\boldsymbol{r}_{i}-\boldsymbol{r}_{k}\right|} .
\end{array}
$$


According to our assumptions the two double sums in (B5) both represent the internal interaction between particles uniformly distributed within a sphere of radius $R$. They must thus be equal, and if we split the Darwin Lagrangian into

$$
L_{\mathrm{mag}}=L_{\mathrm{self}}+L_{\mathrm{int}},
$$

where $L_{\text {self }}$ stands for (B5) and $L_{\text {int }}$ for (B6), we find

$$
L_{\text {self }}=\left(\frac{q_{1}^{2} V_{1}^{2}}{4 c^{2}}+\frac{q_{2}^{2} V_{2}^{2}}{4 c^{2}}\right) \sum_{i} \sum_{j} \frac{1+\cos ^{2} \theta_{i j}}{\left|\boldsymbol{r}_{i}-\boldsymbol{r}_{j}\right|} .
$$

Without the squared cosines the double sum would simply be the Coulomb self interaction of a charged sphere, which can be taken from (A8) with $r=0$. Since the directions of $\boldsymbol{r}_{i}-\boldsymbol{r}_{j}$ vary over the sphere it seems reasonable to estimate the effect of the squared cosine by replacing it with its spherical average

$$
\overline{\cos ^{2} \theta} \equiv \frac{1}{4 \pi} \int_{\Omega} \cos ^{2} \theta \mathrm{d} \Omega=\frac{1}{3},
$$

where $\mathrm{d} \Omega=\sin \theta \mathrm{d} \theta \mathrm{d} \phi$. Also using,

$$
\sum_{i=1}^{N} \sum_{j=1}^{N} \frac{1}{\left|\boldsymbol{r}_{i}-\boldsymbol{r}_{j}\right|}=\frac{6 N^{2}}{5 R}
$$

we get

$$
L_{\text {self }}=\left(\frac{q_{1}^{2} V_{1}^{2}}{4 c^{2}}+\frac{q_{2}^{2} V_{2}^{2}}{4 c^{2}}\right) \frac{4}{3} \frac{6}{5} \frac{N^{2}}{R}=\frac{2 Q^{2}}{5 R c^{2}}\left(V_{1}^{2}+V_{2}^{2}\right),
$$

where, $N q_{1}=Q, N q_{2}=-Q$. We now introduce the transformation (7)-(8). If we put $M_{2} / M \equiv \alpha$ and $M_{1} / M \equiv \beta$ we can write it

$$
\boldsymbol{V}_{1}=\boldsymbol{V}+\alpha \boldsymbol{v}, \text { and } \boldsymbol{V}_{2}=\boldsymbol{V}-\beta \boldsymbol{v},
$$

where $\alpha+\beta=1$. This finally gives

$$
L_{\text {self }}=\frac{2}{5} \frac{Q^{2}}{R c^{2}}\left[2 V^{2}+2(\alpha-\beta) \boldsymbol{V} \cdot \boldsymbol{v}+\left(\alpha^{2}+\beta^{2}\right) v^{2}\right],
$$

for the magnetic self interactions of the spheres.

For the interaction part we put (B12) into (B6) and get,

$$
\begin{array}{r}
L_{\mathrm{int}}=\frac{q_{1} q_{2}}{2 c^{2}}\left[V^{2} \sum_{i, k} \frac{1+\cos ^{2} \theta_{i k}}{\left|\boldsymbol{r}_{i}-\boldsymbol{r}_{k}\right|}-\alpha \beta v^{2} \sum_{i, k} \frac{1+\cos ^{2} \theta_{i k}^{\prime}}{\left|\boldsymbol{r}_{i}-\boldsymbol{r}_{k}\right|}+\right. \\
\left.+(\alpha-\beta) \sum_{i, k} \frac{\boldsymbol{V} \cdot \boldsymbol{v}+\left(\boldsymbol{V} \cdot \boldsymbol{e}_{i k}\right)\left(\boldsymbol{v} \cdot \boldsymbol{e}_{i k}\right)}{\left|\boldsymbol{r}_{i}-\boldsymbol{r}_{k}\right|}\right] .
\end{array}
$$


We now split the vector $\boldsymbol{v}$ into a part parallel to $\boldsymbol{V}$ and a part perpendicular to $\boldsymbol{V}$, to get $\boldsymbol{v}=\boldsymbol{v}_{V}+\boldsymbol{v}_{\perp}$. The double sum in (B15) is then

$$
\sum_{i, k} \frac{\boldsymbol{V} \cdot \boldsymbol{v}+\left(\boldsymbol{V} \cdot \boldsymbol{e}_{i k}\right)\left(\boldsymbol{v} \cdot \boldsymbol{e}_{i k}\right)}{\left|\boldsymbol{r}_{i}-\boldsymbol{r}_{k}\right|}=V v_{V} \sum_{i, k} \frac{1+\cos ^{2} \theta_{i k}}{\left|\boldsymbol{r}_{i}-\boldsymbol{r}_{k}\right|}+V v_{\perp} \sum_{i, k} \frac{\cos \theta_{i k} \cos \theta_{i k}^{\prime \prime}}{\left|\boldsymbol{r}_{i}-\boldsymbol{r}_{k}\right|}
$$

In the above expressions, $\theta_{i k}, \theta_{i k}^{\prime}$, and $\theta_{i k}^{\prime \prime}$, are the angles between $\boldsymbol{r}_{i}-\boldsymbol{r}_{k}$ and $\boldsymbol{V}$, $\boldsymbol{v}$, and $\boldsymbol{v}_{\perp}$, respectively. The double sums are now over points distributed in displaced spheres. If we assume that the displacement $r$ is small compared to the radius $R$ we can again approximate the effect of the cosines by spherical averaging (Bי). We then find that

$$
\overline{1+\cos ^{2} \theta}=\frac{4}{3}, \text { and, } \overline{\cos \theta \cos \theta^{\prime}}=0,
$$

where $\theta$ and $\theta^{\prime}$ represent angles with perpendicular directions.

According to our assumptions we can now use Eq. (A9) and put

$$
\sum_{i, k} \frac{1}{\left|\boldsymbol{r}_{i}-\boldsymbol{r}_{k}\right|} \approx \frac{6 N^{2}}{5 R}-\frac{N^{2} r^{2}}{2 R^{3}}
$$

Finally then we get (note that $V v_{V}=\boldsymbol{V} \cdot \boldsymbol{v}$ )

$$
L_{\mathrm{int}}=-\frac{Q^{2}}{R c^{2}}\left(\frac{2}{5}-\frac{1}{6} \frac{r^{2}}{R^{2}}\right)\left[2 V^{2}+2(\alpha-\beta) \boldsymbol{V} \cdot \boldsymbol{v}-2 \alpha \beta v^{2}\right],
$$

for the magnetic interaction of the two spheres.

If we now add the self interaction, Eq. (B13) to the result (B19) we end up with,

$$
L_{\mathrm{mag}}=\frac{4}{10} \frac{Q^{2}}{R} \frac{v^{2}}{c^{2}}+\frac{1}{3} \frac{Q^{2}}{R} \frac{r^{2}}{R^{2}} \frac{V^{2}+(\alpha-\beta) \boldsymbol{V} \cdot \boldsymbol{v}-\alpha \beta v^{2}}{c^{2}}
$$

for the total magnetic interaction part of the Lagrangian. Here we have used, $\alpha+\beta=1$. Note also that we have assumed that $r \ll R$ and that certain angular dependencies have been treated approximately. If we also assume that $v^{2} \ll c^{2}$ the second term here is negligible compared to the first.

\section{Appendix C: Magnetic interaction of two charged spheres with relative rotation}

If we put $\varrho_{1}=Q / \frac{4 \pi R^{3}}{3}$ and $\varrho_{2}=-\varrho_{1}$, for $r<R$, and zero outside, the two spheres have current densities,

$$
\boldsymbol{j}_{i}(\boldsymbol{r})=\varrho_{i} \boldsymbol{\Omega}_{i} \times \boldsymbol{r}, \quad i=1,2 .
$$


Divergence free vector potentials, that match smoothly with dipole field vector potentials outside the spheres, are then given by [24],

$$
\boldsymbol{A}_{i}(\boldsymbol{r})=\frac{2 \pi}{5 c}\left(\frac{5}{3} R^{2}-r^{2}\right) \boldsymbol{j}_{i}(\boldsymbol{r})
$$

Using this, it is elementary to show that,

$$
L_{\mathrm{mag}}=\frac{1}{2 c} \int \boldsymbol{j}(\boldsymbol{r}) \cdot \boldsymbol{A}(\boldsymbol{r}) \mathrm{d} V=\frac{1}{2 c} \int\left(\boldsymbol{j}_{1} \cdot \boldsymbol{A}_{1}+\boldsymbol{j}_{2} \cdot \boldsymbol{A}_{2}+2 \boldsymbol{j}_{1} \cdot \boldsymbol{A}_{2}\right) \mathrm{d} V,
$$

is given by,

$$
L_{\text {mag }}=\frac{2}{35} \frac{Q^{2} R}{c^{2}} \omega^{2}
$$

where, $\boldsymbol{\omega}=\Omega_{1}-\Omega_{2}$, is the relative angular velocity.

The response to a constant external field $\boldsymbol{B}$ with vector potential $\boldsymbol{A}_{\mathrm{e}}=\frac{1}{2} \boldsymbol{B} \times \boldsymbol{r}$, is given by,

$$
L_{\mathrm{e}}=\frac{1}{c} \int \boldsymbol{j}(\boldsymbol{r}) \cdot \boldsymbol{A}_{\mathrm{e}}(\boldsymbol{r}) \mathrm{d} V=\frac{1}{c} \int\left(\boldsymbol{j}_{1} \cdot \boldsymbol{A}_{\mathrm{e}}+\boldsymbol{j}_{2} \cdot \boldsymbol{A}_{\mathrm{e}}\right) \mathrm{d} V
$$

One finds,

$$
L_{\mathrm{e}}=\frac{Q R^{2}}{10 c} \boldsymbol{B} \cdot \boldsymbol{\omega}
$$

from straightforward calculations.

[1] L. Tonks and I. Langmuir, Phys. Rev. 33, 195 (1929).

[2] D. Bohm and D. Pines, Phys. Rev. 82, 625 (1951).

[3] R. J. Goldston and P. H. Rutherford, Introduction to Plasma Physics (IOP Publishing Ltd, Bristol, 1995), top of page 259.

[4] J. Jellinek and D. H. Li, Phys. Rev. Lett. 62, 241 (1989).

[5] H. Essén, Eur. J. Phys. 14, 201 (1993).

[6] L. D. Landau and E. M. Lifshitz, The Classical Theory of Fields (Pergamon, Oxford, 1975), 4th ed.

[7] C. G. Darwin, Phil. Mag. 39, 537 (1920).

[8] J. D. Jackson, Classical Electrodynamics (John Wiley \& Sons, New York, 1999), 3rd ed.

[9] J. Schwinger, L. L. DeRaad, Jr., K. A. Milton, and W. Tsai, Classical Electrodynamics (Perseus books, Reading, Massachusetts, 1998). 
[10] H. Essén, Phys. Rev. E 53, 5228 (1996).

[11] H. Essén, J. Phys. A: Math. Gen. 32, 2297 (1999).

[12] W. D. Hershberger, J. Appl. Phys. 43, 5204 (1972).

[13] H. Essén, Phys. Rev. E 56, 5858 (1997).

[14] H. Essén and A. B. Nordmark, Phys. Rev. E 69, 036404 (2004).

[15] K. D. Cole, Phys. Plasmas 4, 2072 (1997).

[16] H. Essén, Eur. J. Phys. 26, 279 (2005).

[17] R. M. Kulsrud, Plasma Physics for Astrophysics (Princeton University Press, New Jersey, 2005), pp. 3-4.

[18] J. Frenkel, Phys. Rev. 43, 907 (1933).

[19] H. Alfvén and C.-G. Fälthammar", Cosmical Electrodynamics (Oxford University Press, Oxford, 1963), 2nd ed.

[20] D. C. Barnes and R. A. Nebel, Phys. Plasmas 5, 2498 (1998).

[21] J. Park, R. A. Nebel, S. Stange, and S. K. Murali, Phys. Plasmas 12, 056315 (2005).

[22] D. Lynden-Bell, Phys. Rev. D 70, 104021 (2004).

[23] W. Dobler, M. Stix, and A. Brandenburg, Magnetic field generation in a fully convective rotating sphere, E-print arXiv:astro-ph/0410645 (2004).

[24] H. Essén, Phys. Scr. 40, 761 (1989). 


\section{Figure captions}

FIG. 1: Some notation for the two-sphere plasma model treated in this article. Note the separation of the spheres is exaggerated for clarity. It is assumed small in the relative translation case and zero in the relative rotation case.

FIG. 2: Integration over the upper sphere, centered on $q$, is performed by means of volume elements $\mathrm{d} V=S \mathrm{~d} \rho$ that consist of the space between two concentric sphere segments centered on $Q$ with radii $\rho$ and $\rho+\mathrm{d} \rho$. The area $S$ of such a segment is given by $S=2 \pi \rho h$ where $h=\rho(1-\cos \alpha)$. The cosine theorem applied to the triangle $Q q P$ gives $R^{2}=r^{2}+\rho^{2}-2 r \rho \cos \alpha$. This gives $\cos \alpha$ and insertion into $\mathrm{d} V$ gives $\mathrm{d} V=(\pi \rho / r)\left[R^{2}-(r-\rho)^{2}\right] \mathrm{d} \rho$. 


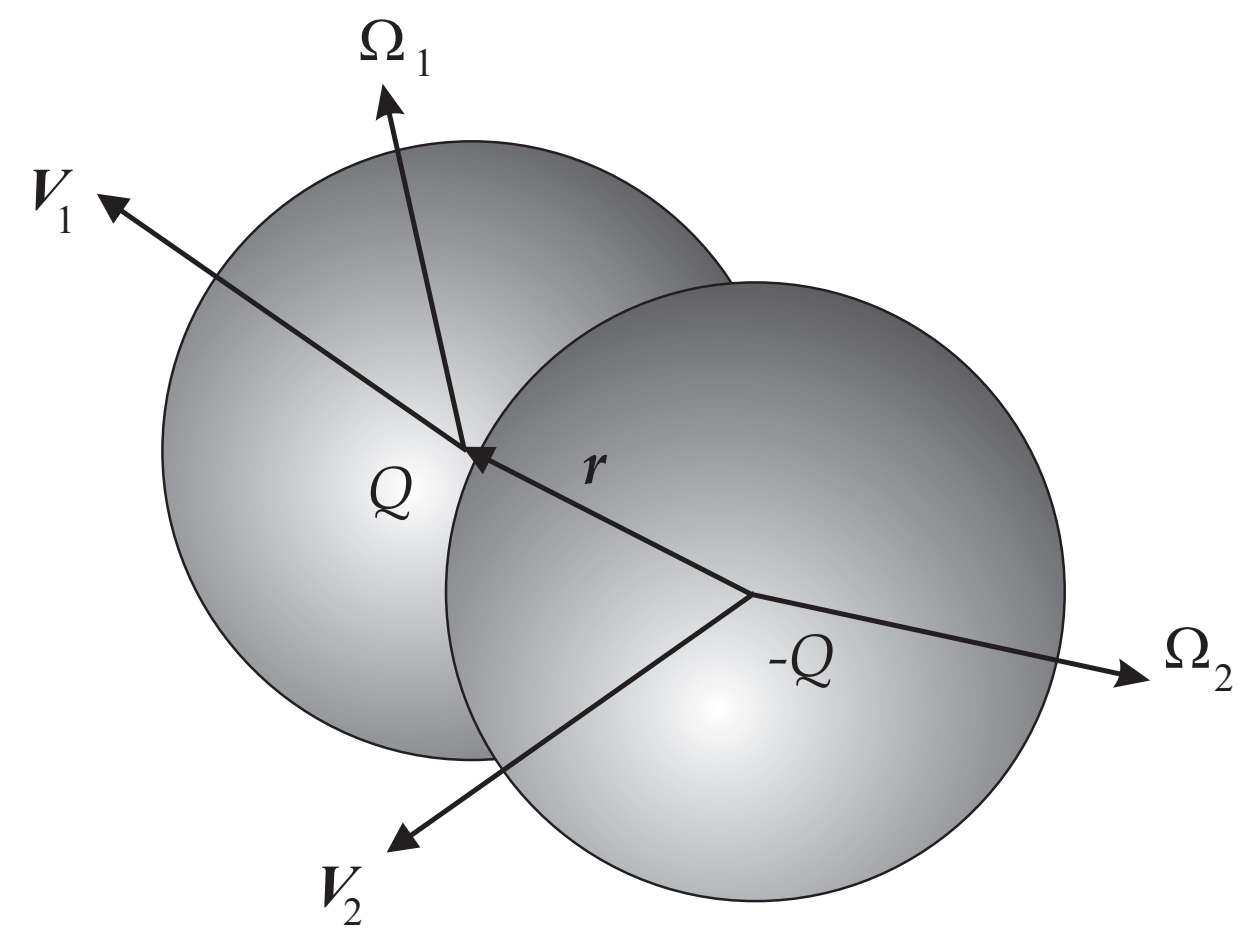




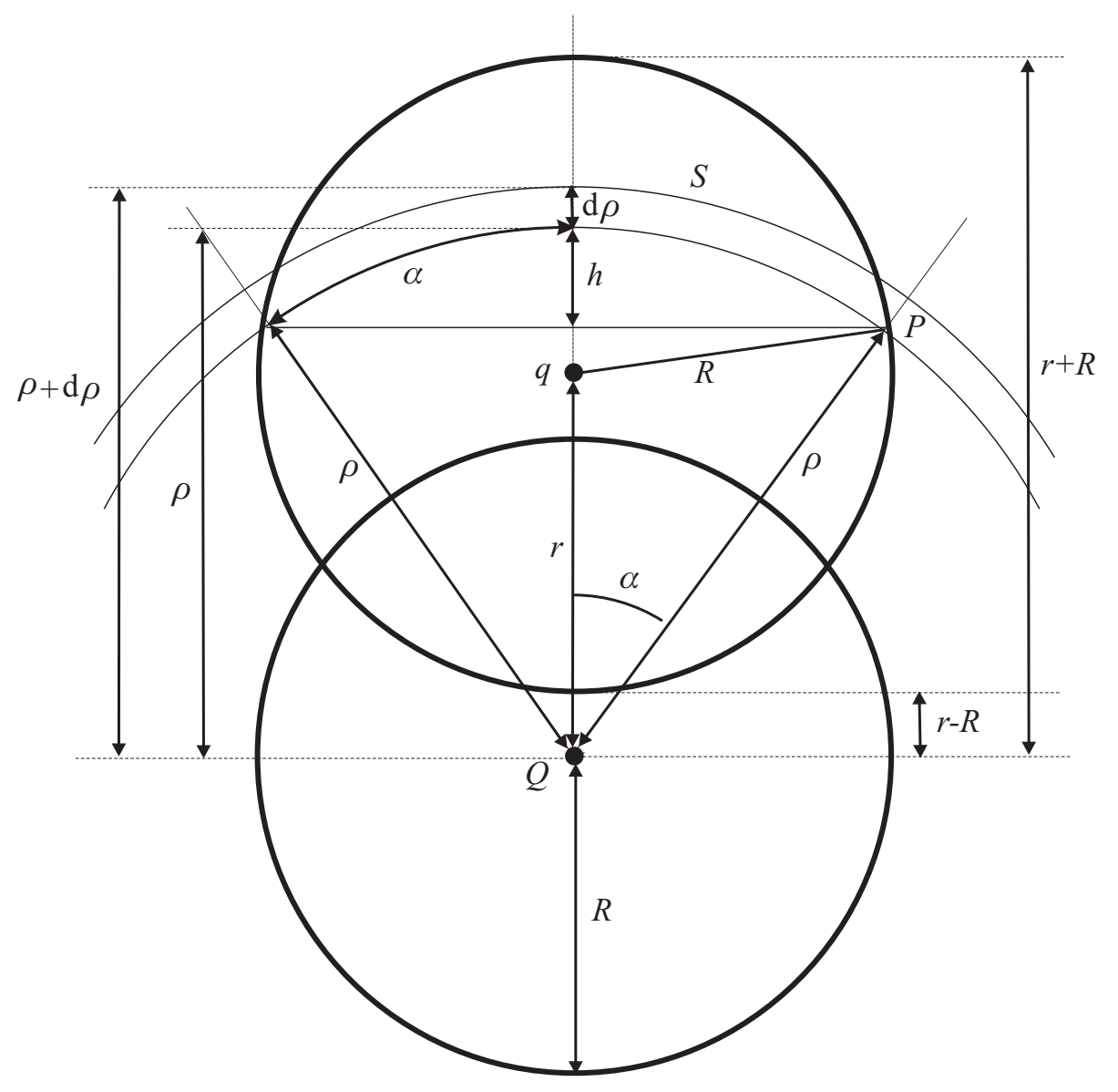

